\title{
Predicted response of Storglaciären, Sweden, to climatic warming
}

\author{
Keith A. Brugger \\ Department of Geology, University of Minnesota-Morris, Morris, MN 56267, U.S.A.
}

\begin{abstract}
A time-dependent model of glacier flow was used to predict the response of Storglaciären, a small valley glacier in northern Sweden, to different warming scenarios by imposing two possible climatic forcings: one in which temperature alone increases ( $\mathrm{T}$ model), and one in which both temperature and precipitation increase (TP model). A range of possible changes in temperature and/or precipitation was related to changes in glacier mass balance through a multiple linear correlation of mean specific net balance with mean summer temperature and mean specific winter balance. The T model was run with mass-balance perturbations in the form of linear increases from the recent (1980-89) mean summer temperature of 1,2 and $4^{\circ} \mathrm{C}$ over the next 100 years. Perturbations for the TP model also used linear increases in precipitation of 10, 20 and $50 \%$ over current mean winter values in addition to increases in temperature.

Results of the modeling suggest that initial changes in the glacier's profile due to increases in temperature, or in both temperature and precipitation, are of comparable magnitude to those that might be expected as the glacier completes its response under the existing climate. Changes in the glacier's surface profile and terminus position that can, with some certainty, be attributed to climatic warming may only become apparent several decades after warming has begun.
\end{abstract}

\section{INTRODUGTION}

Storglaciären is a small sub-polar glacier located in the Kebnekaise massif in northern Sweden (Fig. 1). Annual mass-balance measurements on the glacier began in 1945 (Schytt, 1962) and have continued to the present time. Changes in the glacier's profile, surface elevations and terminus position have also been measured regularly during this period. Analyses of such data led Holmlund (1987, 1988) to conclude that Storglaciären is essentially in equilibrium with the existing climate; that is, only relatively small changes in terminus position and even smaller changes in the ice-surface profile should occur in future in the absence of systematic changes in mass balance. Numerical modeling of Storglaciären's response to the existing climate supports this conclusion (Brugger, 1992). Furthermore, modeling the glacier's response to step-type perturb-ations in mass balance suggests that the volume time-scale $\tau_{\mathrm{v}}$ (defined by Johannesson and others (1989)) as the time required to produce the change in glacier volume between initial and final steady states in response to a perturbation in mass balance) is about 200 years. Because adjustments in glacier geometry caused by perturbations in mass balance are nearly complete after a period of $0.4 \tau_{\mathrm{v}}$ (Jóhannesson and others, 1989), Storglaciären might be expected to be sensitive to $\mathrm{CO}_{2}$-induced warming predicted in many global climate models (e.g. Houghton and others, 1996). Storglaciären's sensitivity could therefore be used to assess in a general way the sensitivity of other similarly sized, valley glaciers. This knowledge of sensitivity is necessary if the behavior of valley glaciers is to be used to detect climatic warming and as a measure of its severity (cf. Oerlemans, 1994; Haeberli and Hoelzle, 1995).

This paper presents results of numerical modeling experiments designed to predict the response of Storglaciären to potential climatic warming. Two general warming scenarios are examined: one in which temperature alone increases and one in which both temperature and precipitation increase. The effects of these increases on the behavior of the glacier are then compared to that predicted in the absence of climate change.

\section{THE MODEL}

The present study uses a time-dependent numerical model developed by Bindschadler $(1978,1982)$ and is only briefly described here. Essentially the model is a one-dimensional, finite-difference approximation to the continuity equation:

$$
\frac{\partial S}{\partial t}+\frac{\partial Q}{\partial x}=b W
$$

where $S$ is the cross-sectional area of the glacier, $t$ is time, $Q$ is the volume flux, $x$ is the center-line distance, $b$ is the annual mass balance, and $W$ is the glacier width. Glacier width and cross-section are parameterized along $x$ as functions of ice thickness $H$ by:

$$
\begin{aligned}
W & =D H^{\frac{1}{2}}+E H \\
S & =\frac{2}{3} D H^{\frac{3}{2}}+\frac{1}{2} E H^{2}+F
\end{aligned}
$$

where $D, E$ and $F$ are constants. Values for $H, W, S, D, E, F$ and the bedrock elevations were determined from detailed maps of the surface of Storglaciären and of the subglacial bedrock topography revealed by radio-echo sounding (Björnsson, 1981). The volume flux is:

$$
Q=f^{*} U_{\mathrm{s}} S
$$

where $f^{*}$ relates the velocity averaged over $S$ to the center- 


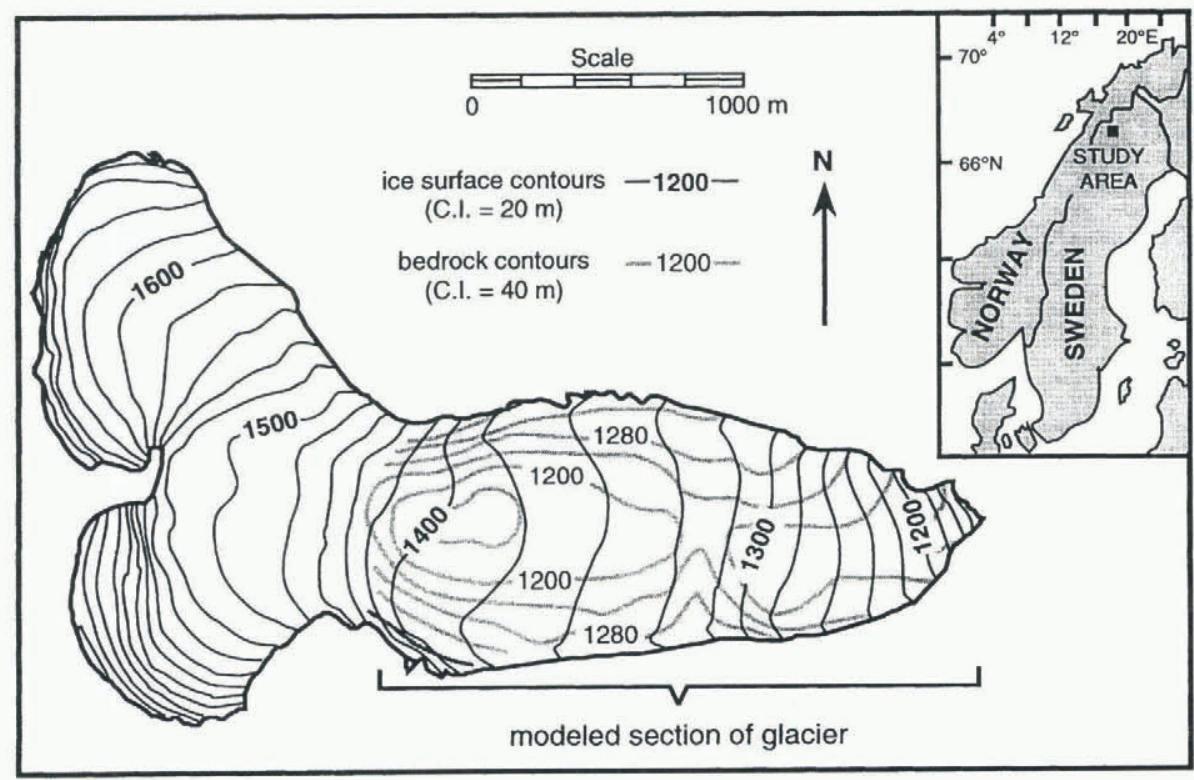

Fig. 1. Map of Storglaciären showing the generalized surface and bed topography. C.I., contour interval.

line surface velocity $U_{\mathrm{s}}$ calculated by:

$$
U_{\mathrm{s}}=\frac{2 A}{n+1}(f \rho g \sin \alpha)^{n} H^{n+1}
$$

where $A$ and $n$ are parameters in a flow law of the form $\dot{\varepsilon}=A \tau^{n}, f$ is a shape factor which accounts for drag imparted by the valley walls, $\rho$ is the density of ice, $g$ is the gravitational acceleration, and $\alpha$ is the slope of the ice surface. Equation (5) ignores any velocity component due to basal sliding, making the model suitable for Storglaciären where velocity data (Hooke and others, 1989; R. LeB. Hooke, unpublished data) indicate that the contributions of sliding to the mean annual velocities do not exceed 5$10 \%$.

Equations (1)-(5) are a set of non-linear simultaneous equations which, when boundary conditions are specified, are solved numerically for the time-dependent behavior of $H$. Details of the computational scheme and its stability can be found in Bindschadler (1978: 84-102). Boundary conditions are specified at the head and terminus of the glacier model. Only the lower part of Storglaciären was modeled, to avoid the complexities of flow dynamics that result from the confluence of the two tributary cirques (Fig. 1). Thus the volume flux into the head of the model must be specified, the details of which are discussed below. The terminus boundary is a wedge in which the geometry varies with time to conserve the volume flux into it, the terminus position being determined where the ice thickness, and hence volume flux, vanish. With all variables, parameterizations and boundary conditions specified, the model was "tuned" by adjusting the $f$ values in Equation (5) so that the calculated surface velocities matched those measured by Hooke and others (1989). The resulting tuned model is the 1985 datum state which is subjected to mass-balance perturbations in the subsequent modeling experiments.

\section{MASS-BALANCE PERTURBATIONS AND CLIMATIC WARMING}

The fundamental influence of climatic warming on the future behavior of Storglaciären is assumed to be that of changes in mass balance brought about by changes in temperature and precipitation. Thus the effect(s) of climatic warming is simulated in the model by imposing various perturbations on the existing mass-balance distribution, defined here as the average during the interval 1981-89. The form and magnitude of the perturbations used are dependent on the presumed increases in temperature and precipitation, and on the relationship between these variables and the mass balance of Storglaciären. The latter relationship for the 43 year period 1946-89 is described by a highly significant correlation (multiple correlation coefficient, $R=0.96$ ) of the variables given by:

$$
\overline{b_{\mathrm{n}}}=-0.394 \overline{T_{\mathrm{s}}}+0.862 \overline{b_{\mathrm{w}}}+0.756
$$

where $\overline{b_{\mathrm{n}}}$ is the mean specific net balance (mw.e.), $\overline{T_{\mathrm{s}}}$ is the local mean summer temperature $\left({ }^{\circ} \mathrm{C}\right)$, and $\overline{b_{\mathrm{w}}}$ is the mean specific winter balance ( $\mathrm{m}$ w.e) that is used here as a surrogate measure of winter precipitation. The magnitude of the mass-balance perturbation $\Delta \overline{b_{\mathrm{n}}}$ for a given change in mean summer temperature $\Delta T$ and/or winter precipitation $\Delta P$ ( $\mathrm{m}$ w.e) is then:

$$
\begin{aligned}
\Delta \overline{b_{\mathrm{n}}}=-0.394\left(\overline{T_{\mathrm{s}(0)}}+\Delta T\right) & +0.862\left(\overline{b_{\mathrm{w}(0)}}+\Delta P\right) \\
+ & 0.756-\overline{b_{\mathrm{n}(0)}}
\end{aligned}
$$

where subscript " 0 " signifies values averaged over the datum interval 1981-89.

The first series of modeling experiments examines the response of Storglaciären to increases in temperature only. These experiments are collectively referred to as the T model. The T-model experiments used mass-balance perturbations (Table l) corresponding to increases of 1,2 and $4^{\circ} \mathrm{C}$ in the present mean summer temperature presumed to increase gradually and linearly over the next 100 years. These increases fall well within the range of those predicted for polar latitudes by recent simulations of greenhouse warming (Houghton and others, 1996). The exact form of the perturbations was that of linear ramp function with a 
Table 1. Mass-balance perturbations

\begin{tabular}{lccc}
\hline Model & $\Delta T^{*}$ & $\Delta P^{*}$ & $\Delta \overline{b_{\mathrm{Il}}}$ \\
& ${ }^{\circ} \mathrm{C}$ & $\%$ & $\mathrm{~m}$ w.e. \\
\hline Existing climate, 1981-89 & 0 & 0 & 0 \\
T model & 1 & 0 & -0.40 \\
& 2 & 0 & -0.80 \\
& 4 & 0 & -1.59 \\
TP model & 1 & 10 & -0.26 \\
& 1 & 20 & -0.12 \\
& 1 & 50 & +0.31 \\
& 2 & 10 & -0.65 \\
& 2 & 20 & -0.51 \\
& 2 & 50 & -0.08 \\
& 4 & 10 & -1.44 \\
& 4 & 20 & -1.30 \\
& 4 & 50 & -0.87 \\
& & &
\end{tabular}

* Increase over 1981-89 values.

limiting value equal in magnitude to $\Delta \overline{b_{\mathrm{n}}}$ at 100 years. The net mass balance $b_{\mathrm{n}}(x, t)$ at any $x$ and time $t$ is thus:

$$
\begin{aligned}
& b_{\mathrm{n}}(x, t)=b_{\mathrm{n}}(x)_{0}+\Delta \overline{b_{\mathrm{n}}} \frac{t}{100} \quad 0 \leq t \leq 100 \\
& b_{\mathrm{n}}(x, t)=b_{\mathrm{n}}(x)_{0}+\Delta \overline{b_{\mathrm{n}}} \quad t>100 .
\end{aligned}
$$

Since the perturbation is applied uniformly over the glacier, this is equivalent to a gradual but continuous translation of the specific net balance curve to a maximum of $\Delta \overline{b_{n}}$, meaning that the mass-balance perturbation is independent of altitude. This seems reasonable since an analysis, following Kuhn (1984), of available specific net balance curves for Storglaciären suggests that this is so, even during years of large positive or large negative mass balance (K. A. Brugger, unpublished data). However, Oerlemans and Hoogendoorn (1989) argue that with climate change the massbalance perturbation might be strongly dependent on altitude; such perturbations are the subject of work in progress.

In the series of experiments examining the effects of increasing both mean summer temperature and winter precipitation (the TP model), a scenario that is predicted by some global climate models, mass-balance perturbations were again applied as a ramp function (Equation (8)). Here, $\Delta \overline{b_{\mathrm{n}}}$ values (Table 1) were calculated (Equation (7)) using precipitation increases of 10, 20 and $50 \%$ over the current winter mean $\left(\overline{b_{\mathrm{w}(0)}}\right)$, together with temperature increases of 1,2 and $4^{\circ} \mathrm{C}$.

The boundary condition at the head of the model must be made to conform to changes in glacier geometry resulting from the mass-balance perturbations. This is somewhat problematic because these changes are unknown in the unmodeled section up-glacier from this boundary. The flux at the head boundary $Q_{\text {head }}(t)$ was allowed to vary according to changes in ice thickness in the following way:

$$
Q_{\text {head }}(t)=\min \left[Q_{\text {head }(0)}\left(\frac{H_{1}(t)}{H_{1(0)}}\right)^{5.5}, Q_{\text {head }(0)}\right]
$$

where $H_{1}(t)$ is the ice thickness at the head, and subscript " 0 " again denotes values averaged over the time interval 1981-89. (The dependence of $Q$ on $H^{5.5}$ arises from Equations (3)-(5) with $n=3$ and the fact that the second term in Equation (3) is small.) The appearance of the minimum function in Equation (9) insures that any initial thickening at $H_{1}$ does not get amplified with time by corresponding increases in $Q_{\text {head. }}$. This would be inconsistent with negative mass-balance perturbations. This restriction was removed for the one experiment in the TP model where $\Delta \overline{b_{\mathrm{n}}}$ was positive (Table 1). $Q_{\text {head }(0)}$ is given by:

$$
Q_{\text {head }(0)}=A_{\mathrm{u}} \overline{b_{\text {nu}(0)}}
$$

where $\overline{b_{\text {nu(0) }}}$ is the mean specific net balance over the unmodeled area $A_{\mathrm{u}}$ up-glacier from the head.

Obviously, a more realistic formulation of $Q_{\text {head }}$ would include a dependence on the surface slope as well, but this requires some knowledge of thickness changes up-glacier from $H_{1}$. Lacking this, Equation (7) assumes that the slope in this region of the glacier remains fairly constant, and the modeling experiments support this assumption. Moreover, $Q$ is less sensitive to changes in slope (see Equation (5)) so that any changes in volume flux brought about by changes in thickness are much greater than those caused by the small changes which occur in the slope.

Because of the uncertainties in this formulation of $Q_{\text {head, }}$, a sensitivity analysis of the model was performed using two alternative formulations. The first required that $Q_{\text {head }}$ decrease in proportion to the imposed mass-balance perturbation such that:

$$
\begin{aligned}
& Q_{\text {head }}(t)=A_{\text {u }}\left(\overline{b_{\text {nu }(0)}}-\Delta \overline{b_{\mathrm{n}}} \frac{t}{100}\right) \quad 0 \leq t \leq 100 \\
& Q_{\text {head }}(t)=A_{\mathrm{u}}\left(\overline{b_{\mathrm{nu}(0)}}-\Delta \overline{b_{\mathrm{n}}}\right) \quad t>100 .
\end{aligned}
$$

In effect this formulation of $Q_{\text {head }}$ implies that the unmodeled region of Storglaciären adjusts instantaneously to the mass-balance perturbations so as to provide a steadystate flux equal to the integral of the net mass balance over the surface $A_{\mathrm{u}}$. This boundary condition insures a monotonic decrease in the volume flux entering the head of the glacier model for the first 100 model years. In the other alternative formulation, $Q_{\text {head }}$ remains constant and equal

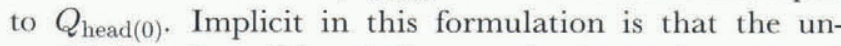
modeled section of the glacier remains in steady state and is unaffected by mass-balance perturbations.

\section{RESULTS AND DISGUSSION}

\section{Predicted behavior in the absence of climate change}

Figure $2 \mathrm{a}$ shows the predicted evolution of the longitudinal profile of Storglaciären assuming no future changes in mass balance. This model will be referred to as the EC model. The EC model thus gives a "baseline" prediction against which results from the Tand TP models can be compared.

The EC model suggests that Storglaciären is quite close to being in steady state with the existing climate. While the terminus continues to adjust over time, most of the glacier experiences only minor changes in geometry (Fig. 2a). Furthermore, Figure 2a suggests that significant changes in terminus geometry will essentially be completed in the next 100 years.

The predicted behavior at the glacier terminus is clarified in Figure 3 where the terminus position is plotted as a function of time. The initial retreat of the terminus from its 1985 position might be unexpected at first, given the glacier's slightly positive specific net balance between 1981 and 1989 (Table 1). However, this behavior continues the trend established around 1910 when the glacier began to re- 

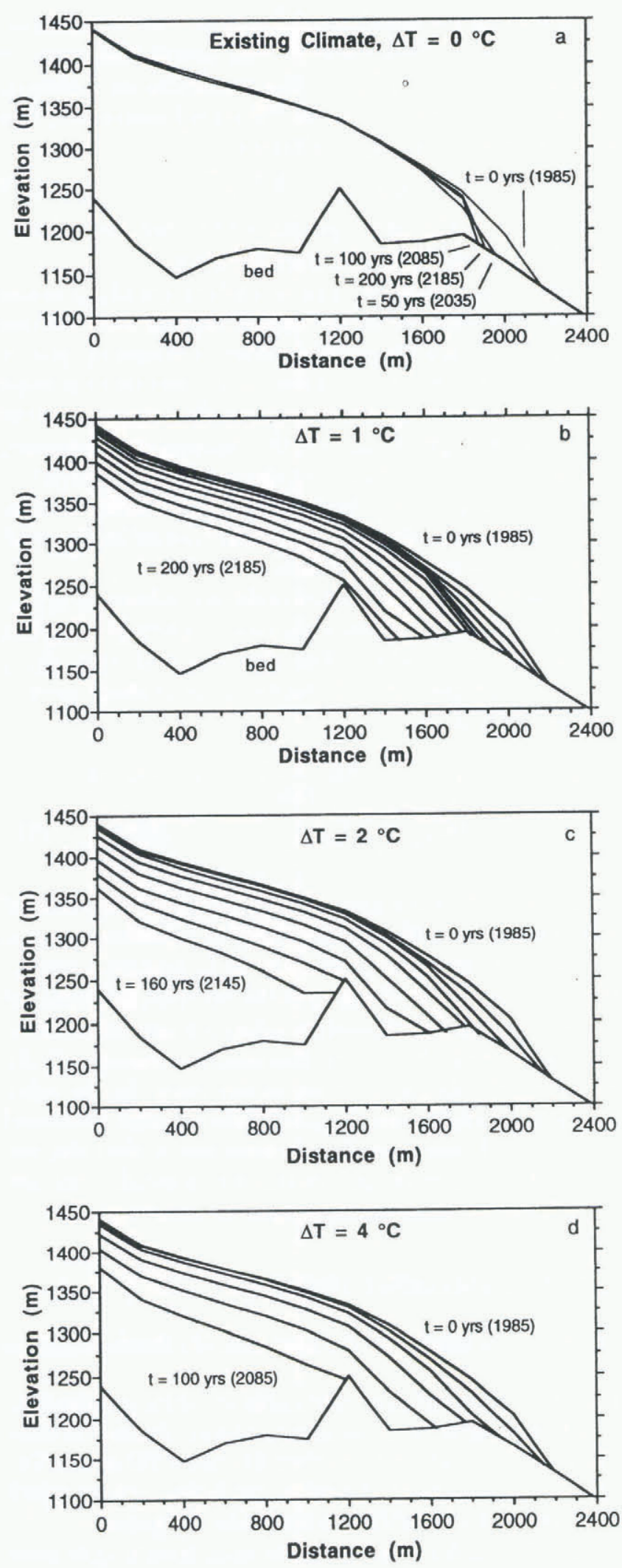

Fig. 2. Evolution of the profile of Storglaciären in response to (a) existing climate (EC model) and $(b-d)$ mass-balance perturbations used in the $T$ model. Profiles are drawn in 20 year intervals except where otherwise labeled.

treat from its Little Ice Age maximum in response to a climatic warming of about $1^{\circ} \mathrm{C}$ (Holmlund, 1987). Thus, the retreat from 1985 to approximately 2070 indicated in Figure 3 reflects the continued adjustment to that warming. Viewed another way, this retreat is a measure of the imbalance between the 1981-89 mass-balance distribution and that required to maintain the existing profile of the glacier.

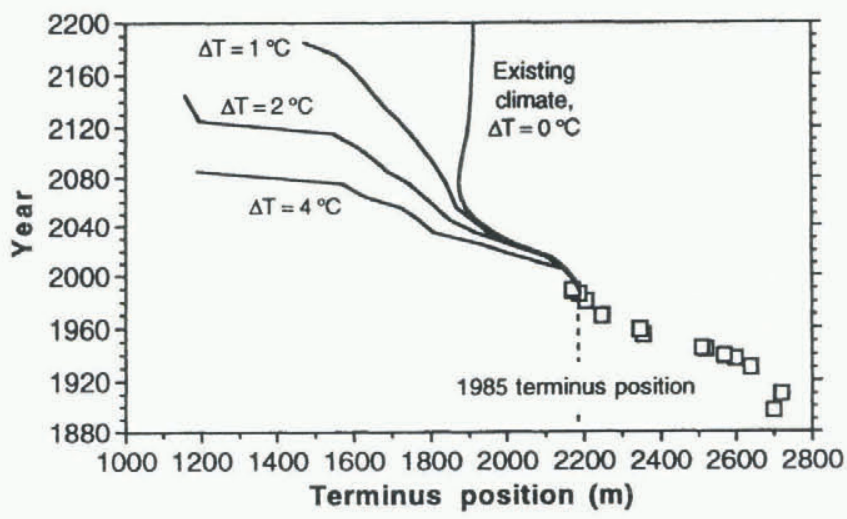

Fig. 3. Predicted behavior of the terminus of Storglaciären in the $T$ model compared with that of the EC model. The squares represent observed terminus positions from 1897 to 1985. The rather rapid retreat from 1550 to $1200 \mathrm{~m}$ for $\Delta T=2^{\circ}$ and $4^{\circ} \mathrm{C}$ is an artifact of retreat over a bedrock riegel.

If the results of the EC model are to be used as the basis for comparison, several points addressing their validity must be emphasized. First, as noted previously, the volume response time $\tau_{\mathrm{v}}$ of Storglaciären has been independently determined to be about 200 years. Adjustments of the terminus in response to the climatic warming in 1910 would therefore be expected to be complete about the year 2110 . In the model, stabilization of the terminus occurs about 2070. These two estimates are in qualitative agreement, considering the uncertainties associated with $\tau_{\mathrm{v}}$ and those inherent in the model. Major changes elsewhere on the glacier should, however, be completed in the much shorter time interval of $0.4 \tau_{\mathrm{v}}$ (Jóhannesson and others, 1989). This means that an equilibrium profile over most of Storglaciären should have been established by 1990 . This is precisely what is seen in the EC model (Fig. 2a) and documented by recent studies of the glacier's mass balance and volume change (Holmlund, 1987, 1988). Thus, the present and future behaviors of the datum glacier according to the EC model are consistent with both observation and theory.

\section{The T model}

The evolution of glacier profiles resulting from the massbalance perturbations introduced in the T model and the corresponding terminus behaviors are shown in Figures $2 \mathrm{~b}-\mathrm{d}$ and 3 , respectively. The objective of each experiment was to simulate the future behavior of Storglaciären for up to 200 model years. For the larger perturbations $\left(2^{\circ}\right.$ and $4^{\circ} \mathrm{C}$ ), however, retreat over a pronounced riegel at $x=1200 \mathrm{~m}$ presented some difficulty in the numerical model as surface slopes became negative immediately upglacier of this point. (See, for example, the 160 year profile for $\Delta T=2^{\circ} \mathrm{C}$ as a precursor of this condition.) These runs were thus terminated early. Also, Figure 2c and d do not show thin residual ice present in the overdeepening immediately down-glacier of the riegel; this ice would presumably stagnate as the active ice front retreats over the riegel.

The most obvious feature of the glacier's response is the reduction in ice thickness, the magnitude of which increases with increasing distance down-glacier. As expected, the reduction in ice thickness scales with the size of the perturbation, and therefore the causal temperature increases. The 


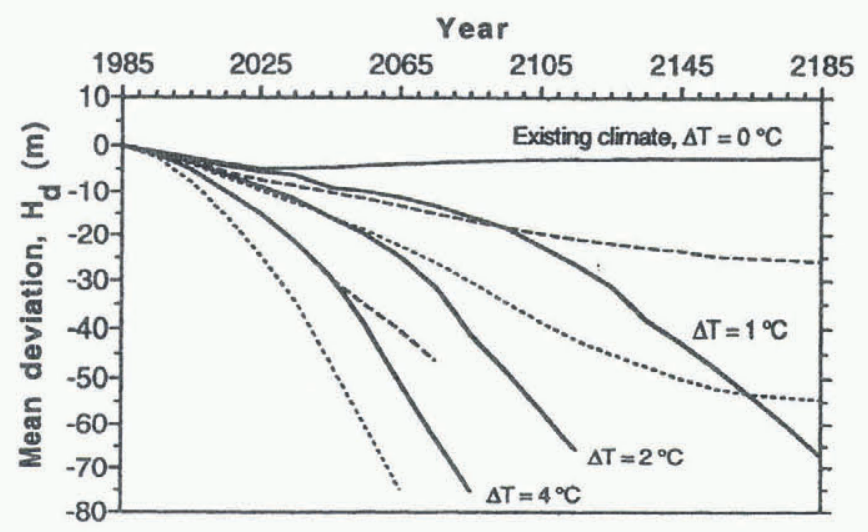

Fig. 4. Mean deviation from the 1985 datum profile for the $T$ model experiments. The upper and lower dashed lines represent results for the constant $Q_{\text {head }}$ boundary condition with $\Delta T=1^{\circ}$ and $4^{\circ} \mathrm{C}$, respectively. The upper and lower dotted lines are for a linearly decreasing $Q_{\text {head }}$ with $\Delta T=1^{\circ}$ and $4^{\circ} \mathrm{C}$, respectively.

mean deviation from the datum profile

$$
H_{\mathrm{d}}(t)=\frac{1}{n} \sum_{i=1}^{n}[H(i, t)-H(i, 1985)],
$$

where $n$ is the number of grid points in the model, is a useful measure when comparing profile changes. $H_{\mathrm{d}}(t)$ for the various modeling experiments are plotted as a function of time in Figure 4. The $H_{\mathrm{d}}(t)$ values of the various $\Delta T$ perturbations are generally not too different from one another nor from those of the EC model in the first 20-30 years of warming (Fig. 4). Even more noteworthy is the fact that $H_{\mathrm{d}}(t)$ for $\Delta T=1^{\circ} \mathrm{C}$ and the EC model are virtually identical for about the first 50 years. These similarities are also evident in the behavior of the terminus (Fig. 3) and in the change in the glacier 's volume (Fig. 5). Only after 70 years do volume changes associated with a warming of $1^{\circ} \mathrm{C}$ begin to deviate significantly from those predicted for the EC model.

Several decades into the warming, however, the massbalance perturbations manifest themselves as relatively large changes in the glacier's terminus position, profile and volume (Figs 3, 4 and 5, respectively). The $H_{\mathrm{d}}(t)$ values vary roughly in proportion to $\Delta T$ so that the effect of the magnitude of $\Delta T$ is now clearly evident. By the end of the warming (2085), the modeled section of Storglaciären has thinned an average of some 16,41 or $75 \mathrm{~m}$ as a result of temperature increases of 1,2 or $4^{\circ} \mathrm{C}$, respectively. This thinning corresponds to a reduction in the glacier's datum-state volume $\left(1.65 \times 10^{8} \mathrm{~m}^{3}\right)$ of between $8 \%\left(1^{\circ} \mathrm{C}\right)$ and $60 \%\left(4^{\circ} \mathrm{C}\right)$. Changes in the glacier's geometry will undoubtedly continue well after 2085 as it continues to adjust to the warmer climate.

Thus, any consequences of climatic warming may not be clearly evident in the short-term behavior of Storglaciären. Rather, such consequences only become observable in the glacier's profile and terminus position 30 or 40 years or more after the onset of warming.

Another characteristic illustrated in Figure $2 \mathrm{~b}-\mathrm{d}$ is that for a given increase in $\Delta t$, the rate of change in ice thickness increases with time. Since the change in ice thickness in these experiments is negative, the rate of change in ice thickness can be thought of as a thinning rate. This behavior appears to be analogous with the initial instability that Nye (1960) showed to be characteristic of the response of the ablation area (or more generally, a zone of compressive flow)

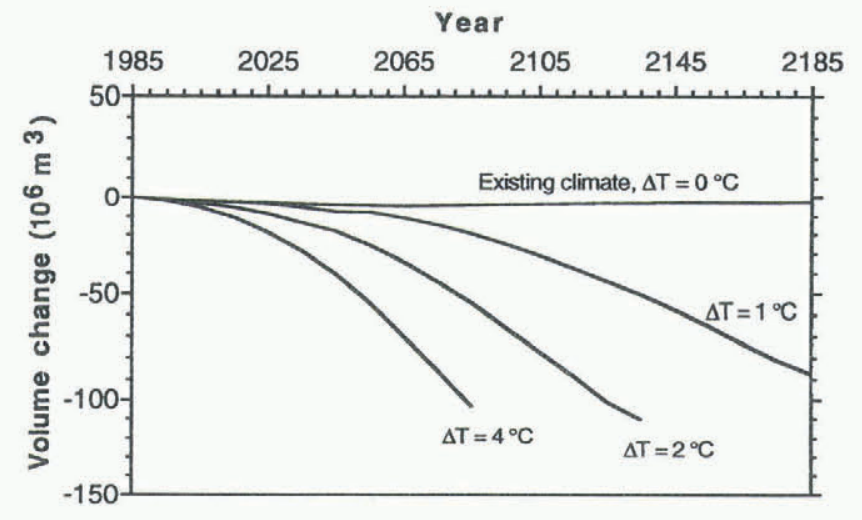

Fig. 5. Volume changes with respect to the 1985 datum profile for the T-model experiments.

to step-type perturbations in mass balance. However, the modeled response of Storglaciären to step-type perturbations is such that any initial instabilities, such as at the terminus, are transient and stability is quickly restored (Brugger, 1992). Thus, the increases in thinning rates observed in the T model are due to the type of perturbation used in the experiments.

\section{Sensitivity analysis of the head boundary condition}

Figure 4 also shows the effects of imposing different headboundary conditions in the $\mathrm{T}$ model using $\Delta T$ 's of $1^{\circ}$ and $4^{\circ} \mathrm{C}$. For an initial period of time, about 110 and 60 years, respectively, a condition of constant $Q_{\text {head }}$ (dashed lines in Fig. 4) yields similar profile changes (quantified in terms of $\left.H_{\mathrm{d}}(t)\right)$ to those when $Q_{\text {head }}$ is calculated from Equation (9). After this time, the $H_{\mathrm{d}}(t)$ values begin to diverge, those associated with the constant flux boundary condition being somewhat smaller in magnitude. The reason for this divergence is the decrease in $Q_{\text {head }}$, insured by Equation (9) by virtue of reductions in $H_{1}$. Eventually the decrease in ice flux becomes large enough that when propagated through the glacier, and when combined with the increasingly negative mass-balance distribution, an increased thinning results. The larger reductions in $H_{1}$ when $\Delta T=4^{\circ} \mathrm{C}$ mean that this occurs sooner than when $\Delta T=1^{\circ} \mathrm{C}$. The condition of constant $Q_{\text {head }}$, although not realistic, places an upper limit on $H_{\mathrm{d}}(t)$ late in the glacier's response, because the ice flux over the upper, unmodeled reaches of Storglaciären will most certainly decrease due to more negative mass balances.

The linearly decreasing $Q_{\text {head }}$ prescribed by Equation (11) (dotted lines in Fig. 4) may place a lower limit on $H_{\mathrm{d}}(t)$. With this boundary condition, $H_{\mathrm{d}}(t)$ shows an initial rate of decrease greater than that either when $Q_{\text {head }}$ is calculated from Equation (9) or when $Q_{\text {head }}$ is held constant. This is because Equation (11) forces a relatively large and rather immediate decrease in $Q_{\text {head }}$, which is not the case for the other two boundary conditions. For the case of $\Delta T=1{ }^{\circ} \mathrm{C}$, the initial rapid decrease in the thickness of the glacier is followed by a period of more gradual decrease, which at 2150 becomes quite small. This transition from an increasing to a decreasing rate of thinning corresponds to the end of the perturbation (100 years), after which $Q_{\text {head }}$ remains constant. (Presumably this would also be seen for $\Delta T=4^{\circ} \mathrm{C}$ if the modeling run could have been continued for the full 200 years.) In view of this, it is not surprising that the rates of thinning for the boundary conditions of constant flux and linearly decreasing flux are similar over the latter halves of the two modeling runs. 


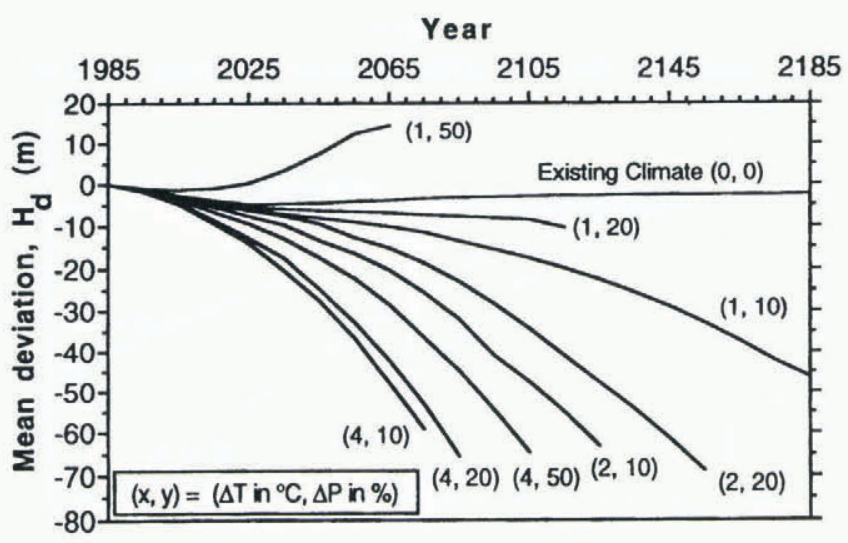

Fig. 6. Mean deviation from the 1985 datum profile for the TP model experiments. The curve for $\Delta T=2^{\circ} \mathrm{C}$ and $\Delta P=$ $50 \%$ is not shown, because of its similarity to that for $\Delta T=1{ }^{\circ}$ Cand $\Delta P=20 \%$ (see Table 1 ).

The foregoing analysis suggests that the behavior of Storglaciären predicted with the boundary condition given by Equation (9) is at least qualitatively correct. Given the uncertainties inherent in the numerical model and in the simulation of the effects of climatic warming on the glacier's mass balance, those introduced by the formulation of the boundary condition are likely to be of lesser significance.

\section{The TP model}

Results of the TP model are shown in Figure 6 as profile changes expressed as variations $H_{\mathrm{d}}(t)$ through time. As in the T model, several of the experiments in the TP model could not be run the full 200 years because of numerical difficulties when the terminus retreats over the riegel. Two of the experiments were terminated early for other reasons. For the case of $\Delta T=1{ }^{\circ} \mathrm{C}$ and $\Delta P=20 \%$, a new steady-state profile was achieved after 134 years. (The term "steady-state" is used here in the context of the numerical model to indicate a condition when profile changes at each grid point fall below a small, predetermined limit.) When $\Delta T=1{ }^{\circ} \mathrm{C}$ and $\Delta P=50 \%$, a rapid advance of the terminus led to numerical difficulties, due in part to the inability to accurately define cross-sectional parameterizations (specifically $f^{*}$ in Equation (4)) down-valley from the present extent of the glacier.

Figure 6 shows that the various $H_{\mathrm{d}}(t)$ curves are indistinguishable from one another and from that for the EC model for the first two decades, again suggesting that the effects of climatic warming may not be evident in the behavior of Storglaciären in the immediate future. For less extreme increases in temperature and/or precipitation these similarities continue for an additional 20-40 years or more.

Because changes in mass balance due to increases in precipitation at least partially offset those due to increases in temperature, thinning of the glacier's profile in each of the TP experiments is less than when only temperature increases are considered. Not surprisingly, this difference becomes more pronounced with increasing values of $\Delta P$. Equation (7) implies that increases in mean summer temperature are wholly offset by increases in winter accumulation when $\Delta P=0.27 \overline{b_{\mathrm{w}(0)}}{ }^{\circ} \mathrm{C}^{-1}$. Thus the combination of $\Delta T=1^{\circ} \mathrm{C}$ and $\Delta P=50 \%$ yields a positive mass-balance perturbation (Table 1) which results in a thickening (Fig. 6) and advance of the glacier. Equation (7) and Figure 6 also suggest that small increases in mean summer temperature $\left(1^{\circ} \mathrm{C}\right)$ and moderate increases in winter accumulation (20$30 \%$ ) could maintain the present, near steady-state geometry of Storglaciären.

\section{GONGLUSIONS}

The foregoing analysis suggests that short-term monitoring of Storglaciären may be insufficient to detect the onset and severity of climatic warming. Initial changes in the glacier's profile and volume predicted in response to increases in temperature or both temperature and precipitation are comparable in magnitude to those that might be expected as the glacier completes its adjustment to the existing climate. Conversely, the lack of rapid, observable changes in the glacier's profile cannot be taken as an indication that climatic warming is not occurring. This ambiguity in the significance of the short-term behavior of Storglaciären is exacerbated with due consideration of: (i) the natural yearto-year variability in mass balance; (ii) past, small-magnitude, high-frequency climatic signals to which the glacier may continue to respond; and (iii) an increase in temperature that may be exponential rather than linear as assumed in this study. Changes in the glacier's surface and terminus position that can, with some certainty, be attributed to climatic warming may only become apparent some 40 years or more after its beginning, depending on the exact nature of the warming. If Storglaciären's sensitivity is typical of small valley glaciers, the above conclusions imply that long-term observations of their behaviors might be required for the detection of climatic warming.

\section{REFERENGES}

Bindschadler, R. A. 1978. Time-dependent model of temperate glacier flow and its application to predict changes in the surge-type Variegated Glacier during its quiescent phase. (Ph.D. thesis, University of Washington.)

Bindschadler, R. 1982. A numerical model of temperate glacier flow applied to the quiescent phase of a surge-type glacier. F. Glaciol., 28 (99), 239-265.

Björnsson, H. 1981. Radio-echo sounding maps of Storglaciären, Isfallsglaciären and Rabots glaciär, northern Sweden. Geogr. Ann., 63A(3-4), 225-231.

Brugger, K. A. 1992. A comparative study of the response of Rabots Glaciär and Storglaciären to recent climatic change. (Ph.D. thesis, Department of Geology and Geophysics, University of Minnesota.)

Haeberli, W. and M. Hoelzle. 1995. Application of inventory data for estimating characteristics of and regional climate-change effects on mountain glaciers: a pilot study with the European Alps. Ann. Glaciol., 21, 206-212.

Holmlund, P. 1987. Mass balance of Storglaciären during the 20th century. Geogr. Ann., 69A(3-4), 439-447.

Holmlund, P. 1988. Is the longitudinal profile of Storglaciären, northern Sweden, in balance with the present climate? 7. Glaciol., 34(118), 269-273.

Hooke, R. LeB., P. Calla, P. Holmlund, M. Nilsson and A. Stroeven. 1989. A 3 year record of seasonal variations in surface velocity, Storglaciären, Sweden. 7. Glaciol., 35(120), 235-247.

Houghton, J. T., L. G. Meiro Filho, B. A. Callander, N. Harris, A. Kattenburg and K. Maskell, eds. 1996. Climate change 1995: the science of climate change. Cambridge, New York, etc., Cambridge University Press. Intergovernmental Panel on Climate Change, WMO/UNEP.

Jóhannesson, T., C. Raymond and E. Waddington. 1989. Time-scale for adjustment of glaciers to changes in mass balance. 7. Glaciol., 35(121), 355-369.

Kuhn, M. 1984. Mass budget imbalances as criterion for a climatic classification of glaciers. Geogr. Ann., 66A(3), 229238.

Nye, J. F. 1960. The response of glaciers and ice-sheets to seasonal and climatic changes. Proc. R. Soc. London, Ser. A, 256(1287), 559-584.

Oerlemans, J. 1994. Quantifying global warming from the retreat of glaciers. Science, 264(5156), $243-245$.

Oerlemans, J. and N. C. Hoogendoorn. 1989. Mass-balance gradients and climatic change. 7. Glaciol., 35(121), 399-405.

Schytt, V. 1962. Mass balance studies in Kebnekajse. 7. Glaciol., 4(33), 281-288. 